\title{
Los asocios público-privados y su alcance en El Salvador
}

El gobierno del presidente Funes ha enviado a la Asamblea Legislativa el anteproyecto de Ley de asocios públicoprivados para su respectiva aprobación. Sin tener a la vista las observaciones, objeciones y apoyos de las diferentes fracciones que conforma esta Órgano del Estado, ya se escuchan pronunciamientos de algunos actores al respecto, tanto de las fracciones que conforman dicho Órgano del Estado, como del sector empresarial y de la sociedad civil.

Examinar la Ley, su contenido y alcance debe de hacerse considerando el contexto económico, político, social y ambiental que define a El Salvador de hoy. No hacerlo de esta forma podría llevar a posiciones ideologizadas y parcializadas, a enfrentamientos estériles, lo que, al final de cuentas, podría beneficiar los intereses de algunos actores, los interesados en su aprobación y puesta en práctica, pero en detrimento de otros sectores.

En todo caso, el análisis de este anteproyecto debe de partir del hecho que ella está concebida como un instrumento de política y no como un fin en sí mismo. Sin la pretensión de agotar el tema, a continuación se presentan algunos planteamientos que tienen la finalidad de aportar a la discusión sobre el alcance de los asocios público-privados como una 
estrategia que en principio busca incentivar la inversión y el crecimiento económico del país.

El desarrollo de El Salvador es un objetivo que atañe al Estado, al sector privado y a la sociedad; cada uno, según sus competencias y funciones. El problema es que ni en su forma filosófica ni en su forma práctica, este objetivo es neutral; en su aplicación se expresan múltiples intereses y relaciones de poder, en el campo de la economía, la política, la cultura, lo social y el medio ambiente.

En el contenido y en la forma que adquiere el "desarrollo" hay una visión paradigmática de la realidad, unos propósitos explícitos e implícitos y unos actores que lo promueven y se benefician más que otros de su puesta en práctica. A pesar de la existencia de algunas prácticas alternativas fundamentadas en otra visión de la realidad, lo cierto es que en el país impera un paradigma dominante de desarrollo intrínsecamente vinculado al sistema capitalista, caracterizado a su vez por un sistema de relaciones de poder asimétrica que promueve y defiende, en función de los intereses de las grandes corporaciones económicas y políticas, el libre mercado, la competitividad, la rentabilidad y la producción de conocimiento. Fuera de esto, estos actores no se movilizan para apoyar otros modos de organización social dirigida a lograr que en los países la población viva bien y en armonía con el medio ambiente. Pareciera que el capitalismo es promocionado como la mejor garantía para lograr el desarrollo humano sostenible.

En lo que respecta al Estado, su rol en las últimas cuatro décadas ha sido garantizar que el sistema capitalista se ocupe, a través del mercado, de impulsar el desarrollo económico, aún a costa de trasladarle al sector privado nacional e internacional sus principales activos mediante procesos de privatización. Luego, con los escasos recursos financieros propios y con la adquisición recurrente de deuda, se ha dedicado a implementar diferentes tipos de políticas públicas; unas dirigidas a mejorar la infraestructura necesaria para la "competitividad"; otras dirigidas a amortizar los impactos negativos de la implementación del modelo de libre mercado y, otras más enfocadas a reducir los efectos de la pobreza estructural. De acuerdo a las estadísticas oficiales, estas políticas han dado algunos resultados. Actualmente se cuenta con un sistema vial moderno, con un aeropuerto moderno, con nuevas instalaciones del sistema nacional de salud o mejoras en algunas de ellas; con varios programas de protección social, por ejemplo. Sin embargo, los avances para reducir las brechas de la exclusión social siguen siendo muy modestos. 
Los tanques de pensamiento asociados al capitalismo han establecido como condición necesaria para lograr el desarrollo que los países incrementen el producto interno bruto, sobre la base de tasas de crecimiento económico superiores al 5\%. El Salvador, bajo la dirección del partido ARENA, llevó a la práctica todo lo que ha estado a su alcance para lograr este objetivo; además de la privatización y de dolarizar la economía, le apostó a los tratados de libre comercio con México, los Estados Unidos y otros muchos países más. Nada de esto incentivó el crecimiento económico. Por el contrario, en promedio, el país no ha logrado superar la barrera de los tres puntos porcentuales en los últimos 25 años. Luego, al examinar año con año el crecimiento económico, aparecen algunos con algún repunte, especialmente en la gestión de Antonio Saca, sin embargo, dicho comportamiento está relacionado fundamentalmente con factores externos y coyunturales, entre los que sobresale el buen precio del café en los mercados internacionales y el incremento de las remesas familiares. Al iniciar la crisis económica mundial y de los Estados Unidos en particular, las tasas de crecimiento descendieron hasta llegar a números negativos.

De la experiencia obtenida a lo largo de estos años se puede concluir que en una gran medida, la apuesta por el crecimiento económico se ha convertido en un fin en sí mismo y no en un medio, entre otros de igual peso, para asegurar el desarrollo del país. Pero también se observa la obstinada posición política de seguir instrumentalizando al Estado en función del crecimiento económico, lo que acarrea importantes implicaciones.

Una de ellas es que se sigue aceptando que el Estado no tiene la capacidad para gerenciar eficientemente los activos que brindan diferentes servicios a la sociedad. Frente a esta ineficiencia, en lugar de trabajar en una nueva estructura y cultura organizacional, se opta por implementar nuevos instrumentos que permitan transferir al sector privado el manejo de estos activos, sin que esto signifique su privatización, bajo la premisa que este sector es más competente para asumir esta tarea. Detrás de esta argumentación y práctica, están presentes la ideología neoliberal y el postulado de que el mercado, "el libre mercado", es capaz de ofrecer bienes y servicios de calidad y a buen precio.

Otra implicación importante es el trato preferencial que el sector empresarial recibe del Estado como parte de los incentivos diseñados para atraer la inversión al país. Más allá de generar empleos, no siempre de calidad, estos manejos terminan por afectar las arcas del Estado, incrementando aún más el déficit fiscal y ante la falta de recursos, desmejorando la capacidad de implementar políticas públicas y debilitando la institucionalidad pública. 
En función del desarrollo inclusivo, el Estado está llamado a crear todas las condiciones que permitan mejorar los procesos de producción de bienes y servicios, teniendo como objetivo la capacidad de incrementar valor a toda la actividad económica. El Estado para cumplir con estas competencias tiene necesariamente que fortalecerse lo que demanda de nuevos procesos de ingeniería institucional que permita el establecimiento de estructuras eficientes y de una cultura organizacional girando en torno a la gestión por resultados. Hipotecar la capacidad del Estado de ofrecer a la sociedad servicios estratégicos que, a su vez, le permiten obtener ingresos para oxigenar las finanzas públicas debe de ser una prioridad de los gobiernos de turno. En aras del crecimiento económico, no sería ético ni políticamente correcto ofrecer al mejor postor del sector privado el usufructo de activos estratégicos para ofrecer bienes y servicios, mucho menos, sería política y económicamente estratégico crear instrumentos de política "a la carta" para atraer inversión privada bajo el pretexto que con ella se logrará estimular el crecimiento económico y con este mejorar el desarrollo del país.

Desde esta óptica, se hace necesario aportar al análisis del proyecto de Ley sobre los Asocios Público-Privados. De acuerdo a la literatura consultada, el fundamento de este tipo de alianzas se base en el establecimiento de acuerdos de colaboración voluntaria, mediante los cuales, el Estado y el privado trabajan conjuntamente para desarrollar una iniciativa específica, compartiendo el financiamiento, el riesgo económico, así como los beneficios dentro de un marco jurídico e institucional en el que no se afecten negativamente los intereses de las partes involucradas (Tennyson, 1998). Por otra parte, el deber ser de este tipo de alianzas es generar un impacto positivo en el desarrollo del país y de los territorios, asegurando un mejor acceso a bienes y servicios necesarios para el bienestar de la sociedad, sin olvidar que la provisión de estos es fundamentalmente una responsabilidad pública, lo que implicaría por parte del Estado que la capitalización de los recursos invertidos por parte del actor privado no sea en detrimento de los consumidores.

En sentido estricto, los asocios público-privados operan mediante el mecanismo de concesión cofinanciada, donde el Estado trasmite a una persona jurídica la construcción, rehabilitación, operación o mantenimiento de una infraestructura o la prestación de un determinado servicio público, durante un plazo de tiempo superior a los 20 años, consignado en unos términos y condiciones acordados y suscritos por ambos actores en un contrato público.

En teoría, los asocios público-privados se han sido diseñados para incrementar la entrega de servicios básicos de infraestructura a la sociedad de 
manera más económica, eficaz y eficiente; a crear opciones de inversión del sector privado en áreas donde son más eficientes; a incrementar la inversión en un mayor número de obras de infraestructura pública, entre otros. En la práctica, la racionalidad económica propia de las empresas y del sector privado tenderá a privilegiar aquellas áreas y sectores que aseguren una mayor rentabilidad y capitalización de las inversiones. Este aspecto, propio del empresariado capitalista, no hay que dejarlo por fuera.

Atendiendo los contenidos del anteproyecto de Ley elaborado por la administración Funes y avalada por el Consejo Económico Social (CES), se hace imperativo hacer algunos planteamientos sobre la finalidad y alcances de esta iniciativa. En primer lugar, llama la atención que el contexto en el que ha nacido esta propuesta, esté marcado por el programa de Asocio para el Crecimiento con Estados Unidos, un pacto para la cooperación en comercio, desarrollo e inversión entre ambas naciones y por un eventual Fomilenio II, que ha recibido por parte de la Corporación del Reto del Milenio (MCC) la aprobación de la propuesta global presentada por el Gobierno de El Salvador para elaborar estudios y análisis de preinversión de los proyectos y para conformar la institucionalidad de la nueva oficina de Fomilenio II, autorizando el desembolso de \$2.9 millones. Más allá de la genuina intención del gobierno de Funes de atraer inversión privada, bajo el pretexto de que esta dinamizará la economía del país, lo cierto es que dicha Ley está en consonancia con ambas iniciativas y con la apuesta agresiva para desarrollar la franja marino costera del país.

Como segundo aspecto, el cual reafirma al anterior, la propuesta de Ley expone que esta será obligatoria para los proyectos y contratos que sean superiores al monto que determina el reglamento, lo que resulta una clara oportunidad para la inversión internacional, en detrimento de la capacidad de inversión nacional, sobre todo de los pequeños y medianos inversionistas. De acuerdo a esta apreciación, los privados con capacidad de invertir, de acuerdo a los montos establecidos, provendrán del extranjero, atraídos, por recibir una rentabilidad adecuada al riesgo que tendrá que asumir y correspondiente a las condiciones del mercado. Según la racionalidad económica que impulsa al sector privado, sólo habrá inversión en aquellos proyectos que los estudios de factibilidad muestren un alto potencial de rentabilidad, lo que puede provocar nuevos desequilibrios en relación al desarrollo de aquellos territorios en desventaja. La rentabilidad económica, bajo esta lógica, será el motor que incentive la productividad, dejando en un segundo plano la rentabilidad social. 
En tercer lugar, en el apartado de los principios generales, la distribución del riesgo tiende a distanciarse de la premisa teórica que justifica la utilización de este instrumento. Se plantea que la parte que esté en mejor posición o tenga mayores competencias para evitar o mitigar los riesgos, deberá de asumir los riesgos. Difícilmente, una empresa privada instalada en el país logrará tener una mejor posición o mayores competencias de las que tiene el Estado, que, a través del gobierno, es el primer interesado en concesionar bajo las modalidades consideradas en el proyecto de Ley algunos de sus activos.

En cuarto lugar, en mismo apartado de los principios generales, se plantea que los participantes privados deberán incorporar y mantener durante toda la fase de ejecución de este tipo de contratos las mejoras prácticas de responsabilidad social empresarial, no obstante, no plantea en relación a que parámetros se evaluará dicha práctica.

En quinto lugar, en el capítulo IV, referido a la aprobación legislativa, queda establecido que cuando dicho proyecto no haya sido aprobado dentro del plazo de cuarenta y cinco días o si esta no aprobara la concesión, el participante privado, tendrá el derecho a recibir a título de indemnización, un monto previamente establecido en las bases de licitación que no podrá exceder el uno por ciento del valor del proyecto. Esta disposición, además que podría poner en apuros al Estado para cumplir con estas obligaciones, puede ser un mecanismo para que el Estado premie aquellos proyectos que desde la visión política de la Asamblea Legislativa, afectaría los intereses nacionales. Lo lógico sería pagarle un porcentaje de los costos invertidos en el proceso de licitación, sin que este sea mayor al cincuenta por ciento, una vez, la entidad que corresponda haya hecho la auditoría correspondiente.

Este anteproyecto de Ley sobre los asocios público-privados, finalmente, tiene como objetivo garantizar las condiciones jurídicas e institucionales para que el sector privado, especialmente el internacional, pueda invertir en el país, de acuerdo a los alcances del Asocio para el Crecimiento con Estados Unidos y con el Fomilenio II. Bajo las condiciones planteadas en este anteproyecto, difícilmente se asegura el desarrollo del país. De hecho, llama la atención que dentro del mismo anteproyecto, en el apartado de los principios generales no se diga nada sobre los derechos laborales, particularmente el derecho a la sindicalización, ni sobre la importancia de generar empleo de calidad, todos estos aspectos, como una condición necesaria para asegurar, desde los intereses laborales, el bienestar y desarrollo del país. 